\title{
Enhanced Charge Fluctuations Due to Competitions between Intersite and Kondo-Yosida Singlet Formations in Heavy-fermion Systems
}

\author{
Kazumasa HATTORI ${ }^{1}$ and Kazumasa MIYAKE ${ }^{2}$ \\ ${ }^{1}$ Institute for Solid State Physics, University of Tokyo, Kashiwa, Chiba 277-8581, Japan \\ ${ }^{2}$ Division of Materials Physics, Department of Materials Engineering Science, Graduate School of Engineering Science, \\ Osaka University, Toyonaka, Osaka 560-8531, Japan
}

\begin{abstract}
We investigate f-electron charge susceptibility in a two-impurity Anderson model on the basis of Wilson's numerical renormalization group method. The f-electron charge susceptibility diverges logarithmically at the critical point of this model when conduction-electron bands exhibit particle-hole symmetry. Although the critical point disappears without the particle-hole symmetry, the f-electron charge fluctuation is much more enhanced near the crossover regime between the Kondo-Yosida singlet and intersite spin-singlet states than that in the singleimpurity case. This result shows that charge fluctuations are enhanced owing to the competition between intersite and Kondo-Yosida spin singlets. A possible scenario for the enhanced residual resistivity near the region where the Kondo temperature becomes comparable with the Néel temperatures under pressure in some heavy-fermion compounds is proposed.
\end{abstract}

KEYWORDS: two-impurity Anderson model, numerical renormalization group, residual resistivity, quantum critical point

Since the first heavy-fermion compound $\mathrm{CeAl}_{3}$ was discovered in $1975,{ }^{1}$ many heavy-fermion f-electron systems have been discovered and investigated. ${ }^{2}$ Many Ce-based compounds show a quantum critical point (QCP) of its long range ordered phase under various external conditions such as pressure and magnetic fields. Near the QCP, anomalous properties appear in many physical quantities such as the diverging specific heat coefficient, the nonFermi liquid (NFL) temperature dependence of the resistivity, and the enhancement in the residual resistivity. Furthermore, an unconventional superconductivity appears near the QCP in many compounds. ${ }^{2}$ Among them, various anomalous properties near the $\mathrm{QCP}$ associated with an antiferromagnetic (AFM) long-range order have attracted much attention. A qualitative explanation of QCPs was proposed by Doniach, ${ }^{3}$ which is competition between the intersite antiferromagnetic order and local Kondo-Yosida singlet formation.

"Conventional" QCPs are well described by the Moriya-Hertz-Millis (MHM)-type critical theory of itinerant magnetism, ${ }^{4-6}$ assuming the existence of quasiparticles and Landau's Fermi liquid theory. ${ }^{7}$ This means that Kondo-Yosida singlet formation starts even inside the AFM phase, and that f-electrons form strongly dressed quasiparticles with a large effective mass in many heavy-fermion compounds. This qualitative picture is the basis of the MHM theory of itinerant AFM fluctuations in heavy-fermion systems.

In some cases such as $\mathrm{CeCu}_{6-x} \mathrm{Au}_{x}{ }^{8}$ with $x=0.1$ and $\mathrm{YbRh}_{2} \mathrm{Si}_{2},{ }^{9}$ the conventional MHM-type critical spin fluctuation theory is not directly applicable, and thus alternative schemes have been proposed, in which changes in the Fermi surface are emphasized. ${ }^{10,11}$ In these theories, no Kondo-Yosida singlet is formed inside the AFM phase. In order to explain the anomalous properties observed in these compounds, Moriya's spin fluctuation theory is also extended. ${ }^{12}$ In spite of these theoretical studies, the anomalous properties of these materials remain to be elucidated.

In this Letter, we will examine the effects of intersite AFM interactions on the formation of heavy quasiparticles and/or Kondo-Yosida singlets in heavy-fermion systems and show that charge fluctuations are strongly enhanced by the formation of Kondo-Yosida singlet states in a two-impurity Anderson model (2IAM).

This result gives a possible explanation for the en-
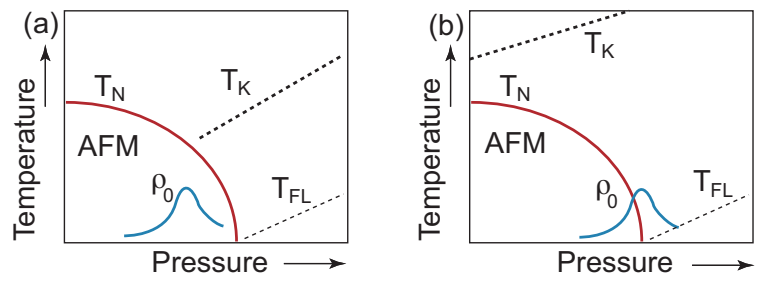

(c)

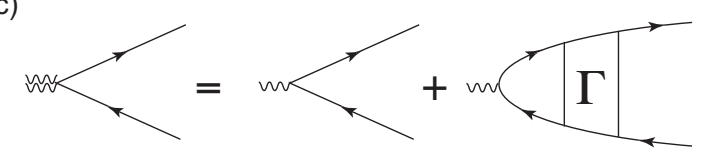

Fig. 1. (Color online) Schematic temperature-pressure phase diagram of Ce-based heavy-fermion systems in the case that $\rho_{0}$ has a peak (a) inside the AFM phase and (b) at the QCP. $T_{\mathrm{FL}}$ is the so-called Fermi liquid temperature. For Yb-based compounds, the horizontal axis should be regarded as a "negative" pressure. (c) Diagrammatic representation of effective potential scattering (double wavy line). The arrows indicate the one-particle Green's function, and the wavy line represents the bare potential scattering. $\Gamma$ is the four-point vertex function. 
hanced residual resistivity inside the AFM phase under pressure in $\mathrm{CeAl}_{2},{ }^{13} \mathrm{CeCu}_{5} \mathrm{Au},{ }^{14}$ and $\mathrm{YbNi}_{2} \mathrm{Ge}_{2},{ }^{15}$ as schematically shown in Fig. 1(a), because nonmagnetic impurity potential is enhanced by charge fluctuations, ${ }^{16}$ leading to the enhanced residual resistivity. Interestingly, the Néel temperature $T_{N}$ and the Kondo temperature $T_{K}$ become comparable at the pressure where the residual resistivity is enhanced. Recently, similar behavior is observed in $\mathrm{CeRu}_{2}\left(\mathrm{Ge}_{x} \mathrm{Si}_{1-x}\right)_{2}$ at approximately $x \sim 0.8 .^{17}$ It is also often the case that the residual resistivity is enhanced at QCP (see Fig. 1(b)). However, in these compounds, the enhanced residual resistivity is observed not at the QCP but inside the AFM phase. This phenomenon has not been well recognized or understood so far, and is interesting because the enhanced residual resistivity cannot be explained by the critical AFM fluctuations near the QCP. ${ }^{18-20}$

As a preliminary step, we review how to estimate, at least qualitatively, the residual resistivity $\rho_{0}$ in heavyfermion systems following the discussions in refs. 18 and 21 ; we focus on the nonmagnetic impurities, which are the dominant sources of the residual resistivity.

First, note that the residual resistivity strongly depends on the effective impurity scattering strength that the quasiparticles are subjected to. One important point is that the quasiparticles mainly consist of f-electrons in heavy-fermion compounds. We will focus on such a situation hereafter. Thus, it is sufficient to take into account only the f-electron component of the quasiparticles. The nonmagnetic impurity scattering strength $u_{\mathbf{q}}$ is renormalized to $\tilde{u}_{\mathbf{q}}$ by the electron-electron interactions, as shown in Fig. 1(c). The residual resistivity is then given as $\rho_{0} \propto N_{F}|\tilde{u}|^{2}$, with the renormalized density of states (DOS) at the Fermi level $N_{F}$. From a detailed analysis of the renormalized $\tilde{u}_{\mathbf{q}}$ on the basis of the Fermi liquid theory in the periodic Anderson model, ${ }^{21}$ it is estimated that $\tilde{u}_{\mathbf{q}} \simeq-u_{\mathbf{q}}\left(d n_{\mathrm{f}} / d \epsilon_{\mathrm{f}}\right) / N_{F}$ in heavy-fermion systems for $\mathbf{q} \rightarrow 0$, where $n_{\mathrm{f}}$ and $\epsilon_{\mathrm{f}}$ are the f-electron number per site and its energy level, respectively. Thus, in heavy-fermion systems, the residual resistivity is enhanced when the f-electron charge susceptibility $\chi_{\mathrm{f}} \equiv-\left(d n_{\mathrm{f}} / d \epsilon_{\mathrm{f}}\right)$ is enhanced.

In this Letter, by using Wilson's numerical renormalization group (NRG) method, ${ }^{22}$ we will investigate the f-electron charge susceptibility $\chi_{\mathrm{f}}$ using 2 IAM as a simplest example, which includes the competition between the Kondo effect and the intersite exchange interactions, and also the charge fluctuations of f-electrons. The model itself has been well understood, ${ }^{23}$ together with a two-impurity Kondo model (2IKM), ${ }^{24-26}$ in the last two decades. There are two stable fixed points. One is the Kondo-Yosida singlet fixed point, where f-electron spin degrees of freedom are screened by those of conduction electrons. The other is an intersite singlet fixed point, where two f-electron spins form a spin-singlet state decoupled from conduction electrons. Between them, there is a NFL fixed point when the conduction electron bands exhibits particle-hole (PH) symmetry. ${ }^{25}$

The Hamiltonian for 2IAM is given by

$$
\begin{gathered}
H=\sum_{\mathbf{k} \sigma} \epsilon_{\mathbf{k}} c_{\mathbf{k} \sigma}^{\dagger} c_{\mathbf{k} \sigma}+\sum_{\sigma \alpha} \epsilon_{\mathrm{f}} f_{\alpha \sigma}^{\dagger} f_{\alpha \sigma}+U \sum_{\alpha} f_{\alpha \uparrow}^{\dagger} f_{\alpha \uparrow} f_{\alpha \downarrow}^{\dagger} f_{\alpha \downarrow} \\
+\frac{v}{\sqrt{N_{0}}} \sum_{\mathbf{k} \sigma \alpha}\left[e^{i \mathbf{k} \mathbf{x}_{\alpha}} f_{\alpha \sigma}^{\dagger} c_{\mathbf{k} \sigma}+\text { h.c. }\right]+J \mathbf{S}_{1} \cdot \mathbf{S}_{2},
\end{gathered}
$$

where $f_{\alpha \sigma}^{\dagger}$ is an f-electron creation operator with spin $\sigma=\uparrow$ or $\downarrow$ and site index $\alpha=1$ or 2 , and $\mathbf{S}_{\alpha}$ is a spin operator of an f-electron at postion $\mathbf{x} \alpha \cdot c_{\mathbf{k} \sigma}^{\dagger}$ is a conduction electron creation operator with wave number $\mathbf{k}$ and spin $\sigma . N_{0}, \epsilon_{\mathbf{k}}, U$ and $v$ are the number of sites, the kinetic energy of conduction electrons, and the Coulomb repulsion and hybridization, respectively. $J$ is a phenomenological intersite exchange interaction between f-electrons and it is assumed to be antiferromagnetic, $J>0$. This is introduced to enlarge the parameter space of 2IAM. One of the physical interpretations of $J$ is, for example, the superexchange interaction due to the direct hopping processes of f-electrons. For later purposes, we define the distance between the two sites as $R \equiv\left|\mathbf{x}_{1}-\mathbf{x}_{2}\right|$. We set $U=10 D$ ( $D$ being half the bandwidth of conduction electrons) and vary $\epsilon_{\mathrm{f}}, v$, and $J$ to examine the variations of $\chi_{\mathrm{f}}$.

In the NRG calculation, we transform eq. (1) into a form of semi-infinite one-dimensional chains, and diagonalize it iteratively. $^{22}$ In the two-impurity model, we have two conduction electron bands, namely, even and odd bands reflecting the mirror symmetry of the system. ${ }^{24}$ We use the logarithmic discretization parameter $\Lambda=3$ and the low-energy 1000 states are kept at each NRG iteration.

First, we discuss the case of the $\mathrm{PH}$-symmetric conduction electron bands. We use a constant DOS for both the even and odd conduction electrons in the following. In the final part of this Letter, we will examine the effects of $\mathrm{PH}$ asymmetry.

Figure 2(a) shows the total f-electron number $N_{\mathrm{f}} \equiv$ $\sum_{\sigma \alpha}\left\langle f_{\alpha \sigma}^{\dagger} f_{\alpha \sigma}\right\rangle$ for $\epsilon_{\mathrm{f}}=-0.8 D$ and $v=0.18 D$ as a function of $J-J_{c}$, where $\langle\cdots\rangle$ indicates the thermal average and $J_{c}$ is the critical value of $J, J_{c}=0.0018215 D$, for this parameter set. At high temperatures $T, N_{\mathrm{f}}$ gradually increases as $J$ increases, while the variation becomes steeper near $J=J_{c}$ as $T$ decreases. Corresponding to this, $\chi_{\mathrm{f}}$ has a sharp peak at $J=J_{c}$ as a function of $J$ at low temperatures, as shown in Figs. 2(b) and (c).

Figure $2(\mathrm{c})$ shows that the $J-J_{c}$ dependence of $\chi_{\mathrm{f}}(0)$ is logarithmic, i.e.,

$$
\chi_{\mathrm{f}}(0) \propto-\log \left|J-J_{c}\right| \text { for }\left|J-J_{c}\right| / J_{c} \ll 1,
$$

at $T=0$. As for the temperature dependence of $\chi_{\mathrm{f}}, \chi_{\mathrm{f}}(T)$ just at the critical point is shown in Fig. 3 for three values of $\epsilon_{\mathrm{f}}$. Note that the charge fluctuation is enhanced as $\left|\epsilon_{\mathrm{f}}\right|$ decreases and $\chi_{\mathrm{f}}$ increases with decreasing $T$ and diverges at $T=0$. As shown in Fig. 3, the temperature 

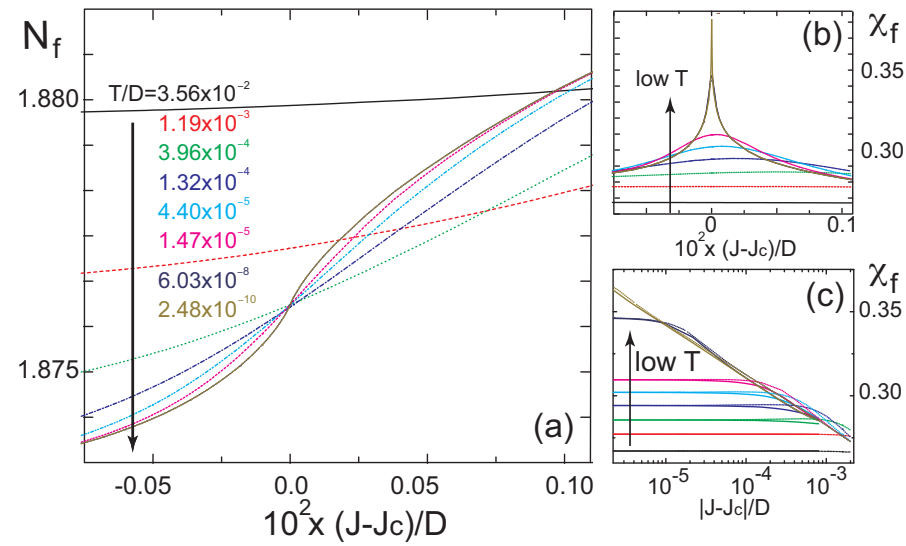

Fig. 2. (Color online) (a) Total f-electron number $N_{\mathrm{f}}$ vs $J-J_{c}$ for eight different temperatures for $v / D=0.18$ with $\mathrm{PH}$-symmetric conduction electron bands. (b) f-electron charge susceptibility $\chi_{\mathrm{f}}(T)$ vs $J-J_{c}$. (c) $\chi_{\mathrm{f}}(T)$ for $J-J_{c}<0$ (dotted lines) and $J-J_{c}>0$ (full lines) on logarithmic scale.

dependence of $\chi_{\mathrm{f}}(T)$ is also logarithmic:

$$
\chi_{\mathrm{f}}(T) \propto-\log T \quad \text { for } J=J_{c} .
$$

Corresponding to the diverging f-electron charge susceptibility at $J=J_{c}$, the imaginary part of the dynamical f-electron charge susceptibility $\operatorname{Im} \chi_{\mathrm{f}}(\omega)$ has a finite amplitude at the frequency $\omega=0$ and at $J=J_{c}{ }^{27}$ where a finite residual entropy $\log \sqrt{2}$ remains. ${ }^{28}$ Away from the critical point, $\operatorname{Im} \chi_{\mathrm{f}}(\omega)$ is proportional to $\omega$ at low frequency, recovering the Fermi liquid behavior.

The above results suggest that there is a singularity in the charge sector through the formation of the KondoYosida singlet between the $\mathrm{f}$ - and conduction electrons leading to a steep change in $N_{\mathrm{f}}$. The diverging $\chi_{\mathrm{f}}$ is related to the steep change in the phase shift between $\delta=0$ and $\pi / 2$ in the case of the PH-symmetric conduction electrons. In order to explain this, it is essential to note that the numbers of f-electrons are different between the two fixed points: the f-electrons are more localized in the inter-site spin-singlet fixed point than in the KondoYosida singlet fixed point, and the singular $\left|J-J_{c}\right|$ dependence in $\chi_{\mathrm{f}}$ appears between these two stable fixed points, i.e., at the NFL fixed point.

On the basis of the boundary conformal field theoretical approach in $2 \mathrm{IKM},{ }^{25}$ it is expected that the spinsinglet Cooper channel and the charge susceptibility of the conduction electrons diverge at the NFL fixed point. It is often the case that the charge susceptibility of conduction electrons diverges at NFL fixed points in various impurity models, such as the multichannel ${ }^{28}$ and spin $3 / 2$ multipolar ${ }^{29}$ Kondo models.

The origin of the logarithmic divergence $\chi_{\mathrm{f}}(T) \propto$ $-\log T$ is related to the existence of boundary operators with the scaling dimension $\Delta=1 / 2$ near the critical point of 2IAM. Here, we assume that the operator contents at the critical point of the 2IAM is the same

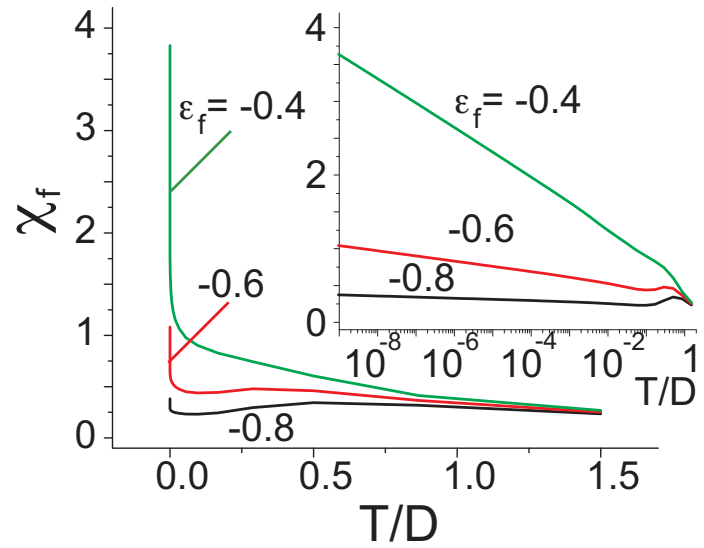

Fig. 3. (Color online) Temperature dependence of the f-electron charge susceptibility $\chi_{\mathrm{f}}(T)$ at $J=J_{c}\left(\epsilon_{\mathrm{f}}\right)$ for $\epsilon_{\mathrm{f}} / D=-0.4,-0.6$ and -0.8 , and $v / D=0.18$ with PH-symmetric conduction electron bands. Inset: $\chi_{\mathrm{f}}(T)$ on logarithmic temperature scale.

as that in $2 \mathrm{IKM}$, which is a reasonable assumption. The candidate is the charge vector field operator $\vec{\phi}_{c}$ in Table VI in ref. 25. $\vec{\phi}_{c}$ can be regarded as the f-electron charge operator and can couple with the first descendant of the total charge current operator $\mathbf{J}_{-1}^{c}$ around the NFL fixed point.

Following the discussions in the multichannel Kondo model, ${ }^{28}$ we obtain the logarithmic temperature dependence of the charge susceptibility. We also obtain logarithmic divergence in the specific heat coefficient. As discussed by Johannesson et al., in the context of the two-channel Anderson model, ${ }^{30}$ the important point is that the coefficient in front of the logarithm is expected to be scaled by $1 / D$ rather than by $1 / T_{K}$, leading to a small coupling constant. Then, we can safely neglect it in the specific heat calculation. However, in the calculation of the charge susceptibility, we cannot neglect it, since there is no contribution of other operators here. This small coefficient is the reason why we find moderate divergence in the f-electron charge susceptibility in Figs. 2 and 3.

In order to discuss a more realistic situation, we introduce $\mathrm{PH}$ asymmetry to the conduction electrons below. The importance of the PH symmetry in 2IKM and 2IAM has been studied in detail. ${ }^{23,25}$ Indeed, the model lacks the critical point present in the PH-symmetric case. The two different fixed points with phase shifts $\delta=0$ and $\pi / 2$ can be connected by a smooth crossover. Although it is not a transition but a crossover, the nonmonotonic variation in $\chi_{\mathrm{f}}$ is expected owing to the "hidden" NFL fixed point. In the numerical calculations below, we set $\epsilon_{\mathbf{k}} \simeq v_{F}\left(k-k_{F}\right), k_{F} R=\pi$ and $D=v_{F} k_{F} / \pi$, where $v_{F}$ and $k_{F}$ are the Fermi velocity and momentum, respectively. For these parameters, an intersite exchange interaction is induced, which is antiferromagnetic. ${ }^{26}$ Thus, we set $J=0$. The density of states $\rho_{ \pm}$for the even $(+)$ and odd ( - ) bands of conduction electrons are given as 


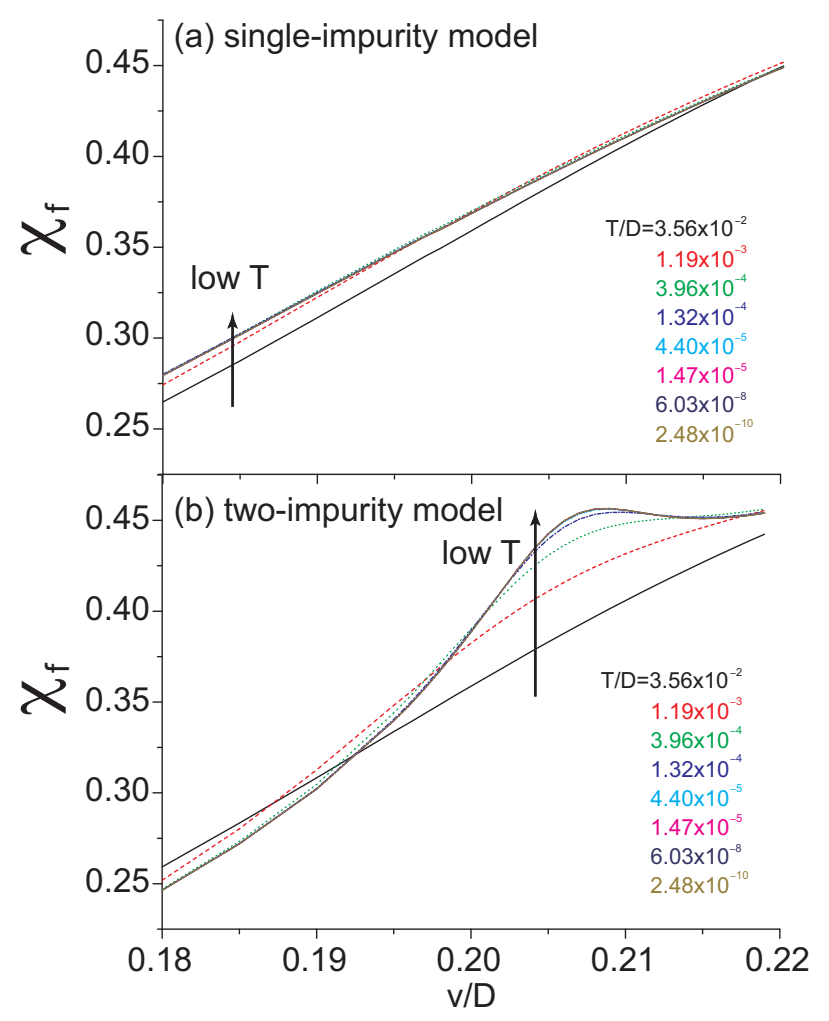

Fig. 4. (Color online) f-Electron charge susceptibility $\chi_{\mathrm{f}}(T)$ vs $v$ for $\epsilon_{\mathrm{f}} / D=-0.8, J=0$. (a) Single impurity model with the constant DOS, and (b) two-impurity model without PH symmetry. For (a), the value is multiplied by the factor 2 .

$\rho_{ \pm}=A_{ \pm}[1 \pm(\sin k R) / k R]$, where $A_{ \pm}$is the normalization constant. ${ }^{31}$

Figure 4 shows the $v$ dependence of $\chi_{\mathrm{f}}$ for eight different temperatures for (a) the single-impurity Anderson model with a constant DOS and (b) 2IAM. As expected, $\chi_{\mathrm{f}}(T)$ for the single-impurity model shows a monotonic increase as $v$ increases in (a). This is natural since as $v$ increases, the charge fluctuations of f-electrons are enhanced. In the case of the 2IAM, as shown in (b), $\chi_{\mathrm{f}}(T)$ develops a peak structure at low temperatures at approximately around $v \sim 0.205 D$ in addition to the monotonic increase as $v$ increases. Around $v=0.205 D$, the KondoYosida singlet starts to form as $v$ increases, while for $v \lesssim 0.205 D$ two f-electrons form a spin singlet owing to the induced antiferromagnetic interaction.

As discussed above, we have demonstrated that $\chi_{\mathrm{f}}$ is enhanced near the critical point and the crossover region between the Kondo-Yosida singlet and the intersite spin-singlet states. By combining this result with those in refs. 18 and 21, it is expected that the residual resistivity is enhanced owing to the Kondo-Yosida singlet formation. Although our analysis is based on a two-impurity problem, we expect that the same phenomenon exists in the periodic lattice models for heavy-fermion systems. More elaborate calculations are necessary to examine the present scenario for the enhanced residual resistivity in heavy-fermion systems.

As for the Fermi surfaces in the Anderson lattice model, the occurrence of the Kondo-Yosida singlet formation strongly affects the topology of the Fermi surfaces inside the AFM phase. From this point, it is expected that the residual resistivity anomaly will have a close relation with the changes in the Fermi surface. In real materials, as shown in Fig. 1(a), the system is inside the AFM phase at low temperatures when the residual resistivity shows enhancement as a function of pressure. In the AFM phase, the topology of Fermi surfaces in the Anderson lattice model is not yet fully understood and it is also nontrivial to regard it to be the same as that in the Kondo lattice model. A recent variational Monte Carlo study shows the existence of two different phases in the AFM phase. ${ }^{32}$ Further theoretical studies are necessary to clarify the relationship between the Kondo-Yosida singlet formation and the enhanced residual resistivity.

In summary, we have discussed the f-electron charge susceptibility $\chi_{\mathrm{f}}(T)$ near the critical point of the two-impurity Anderson model. We have found that $\chi_{\mathrm{f}}(T)$ diverges at the critical point of this model with particle-hole-symmetric conduction electrons. Even when the conduction electron bands are not particlehole-symmetric and thus the critical point smears out, $\chi_{\mathrm{f}}(T)$ is still much more enhanced than that in the single-impurity case around the crossover regime between the Kondo-Yosida singlet and intersite spin-singlet fixed points. These results shed light on the effects of intersite correlation on heavy-fermion formation and the enhanced residual resistivity in Ce- and Yb-based heavyfermion compounds.

\section{Acknowledgments}

K. H. acknowledge useful discussions with C. M. Varma and L. Zhu. K. H. also acknowledge hospitality from the University of California, Riverside, while part of this work was done. This work was supported by a Grant-in-Aid for Scientific Research (No. 20740189) from the Japan Society for the Promotion of Science, and by a Grant-in-Aid for Scientific Research in Innovative Areas "Heavy Electrons" (No. 20102008) from the Ministry of Education, Culture, Sports, Science and Technology, Japan.

1) K. Andres, J. E. Graebner, and H. R. Ott: Phys. Rev. Lett. 35 (1975) 1779.

2) G. R. Stewart: Rev. Mod. Phys. 56 (1984) 755.

3) S. Doniach: Physica (Amsterdam) 91B+C (1977) 231.

4) T. Moriya: "Spin Fluctuations in Itinerant Electron Magnetism", (Springer-Verlag, Berlin, 1985).

5) J. A. Hertz: Phys. Rev. B 14 (1976) 1165.

6) A. J. Millis: Phys. Rev. B 48 (1993) 7183.

7) L. D. Landau: Zh. Eksp. Teor. Fiz. 30 (1956) 1058 [Translation: Sov. Phys. JETP 3 (1957) 920].

8) A. Schröder, G. Aeppli, R. Coldea, M. Adams, O. Stockert, H. v. Löhneysen, E. Bucher, R. Ramazashvili, and P. Coleman: Nature 407 (2000) 351. 
9) O. Trovarelli, C. Geibel, S. Mederle, C. Langhammer, F. M. Grosche, P. Gegenwart, M. Lang, G. Sparn, and F. Steglich: Phys. Rev. Lett. 85 (2000) 626.

10) P. Coleman, C. Pépin, Q. Si, and R. Ramazashvili: J. Phys.: Condens. Matter 13 (2001) R723.

11) Q. Si, S. Rabello, K. Ingersent, and J. L. Smith: Nature 413 (2001) 804.

12) T. Misawa, Y. Yamaji, and M. Imada: J. Phys. Soc. Jpn. 78 (2009) 084707.

13) B. Barbara, J. Beille, B. Cheaito, J. M. Laurant, M. F. Rossignol, A. Waintal, and S. Zemirli: J. Physique (Paris) 48 (1987) 635.

14) H. Wilhelm, S. Raymond, D. Jaccard, O. Stockert, H. v. Löhneysen, and A. Rosch: J. Phys.: Condens. Matter 13 (2001) L329.

15) G. Knebel, D. Braithwaite, G. Lapertot, P. C. Canfield, and J. Flouquet: J. Phys.: Condens. Matter 13 (2001) 10935.

16) O. Betbeder-Matibet and P. Noziéres: Ann. Phys. 37 (1966) 17.

17) Y. Matsumoto: private communications.

18) K. Miyake and O. Narikiyo: J. Phys. Soc. Jpn. 71 (2002) 867.

19) H. Kontani and M. Ohno: Phys. Rev. B 74 (2006) 014406.
20) In the case shown in Fig. 1(b), there also remains the possibility that the charge fluctuations associated with the KondoYosida singlet formation at the QCP enhances residual resistivity.

21) K. Miyake and H. Maebashi: J. Phys. Soc. Jpn. 71 (2002) 1007.

22) K. G. Wilson: Rev. Mod. Phys. 47 (1975) 773.

23) O. Sakai, Y. Shimizu, and T. Kasuya: Solid State Commun. 75 (1990) 81.

$24)$ B. A. Jones and C. Varma: Phys. Rev. Lett. 58 (1987) 843.

25) I. Affleck, W. W. Ludwig, and B. A. Jones: Phys. Rev. B 52 (1995) 9528.

26) J. B. Silva, W. L. C. Lima, W. C. Oliveira, J. L. N. Mello, L. N. Oliveira, and J. W. Wilkins: Phys. Rev. Lett. 76 (1996) 275.

27) K. Hattori and K. Miyake J. Magn. Magn. Mat. 310 (2007) 452.

28) I. Affleck and W. W. Ludwig: Nucl. Phys. B360 (1991) 641.

29) K. Hattori: J. Phys. Soc. Jpn. 74 (2005) 3135.

30) H. Johannesson, N. Andrei, and C. J. Bolech: Phys. Rev. B 68 (2003) 075112.

31) D. L. Cox and A. Zawadowski: Adv. Phys. 47 (1998) 599.

32) H. Watanabe and M. Ogata: J. Phys. Soc. Jpn. 78 (2009) 024715 . 